\title{
Cost-Effectiveness Analysis of Adjuvant Neratinib Following Trastuzumab in Early-Stage HER2-Positive Breast Cancer
}

\author{
Naomi R.M. Schwartz, MPH; Meghan R. Flanagan, MD, MPH; Joseph B. Babigumira, PhD, MS;
} Lotte M. Steuten, PhD, MSc; and Joshua A. Roth, PhD, MHA

\begin{abstract}
BACKGROUND: Disease-free survival (DFS) in early-stage human epidermal growth factor receptor 2 (HER2)-positive breast cancer is significantly greater with the addition of neratinib after adjuvant trastuzumab versus no additional therapy. However, it remains uncertain whether these survival gains represent good value for the money, given the substantial cost of neratinib.

OBJECTIVE: To evaluate clinical and economic implications of adding neratinib after adjuvant trastuzumab based on results from the phase III ExteNET trial.

METHODS: A 3-state Markov model was developed to estimate the costeffectiveness of neratinib for women with early-stage (I-III) HER2-positive breast cancer. Five-year recurrence rates were derived from the ExteNET trial. Mortality and recurrence rates after 5 years were derived from the HERceptin Adjuvant (HERA) trial. Outcomes included life-years, qualityadjusted life-years (QALYs), and direct medical expenditures. The analysis was performed from a payer perspective over a lifetime horizon. One-way sensitivity and probabilistic analyses were conducted to evaluate uncertainty.

RESULTS: Total cost of neratinib following adjuvant trastuzumab was $\$ 317,619$ versus $\$ 152,812$ for adjuvant trastuzumab alone. A gain of 0.4 QALYs (15.7 vs. 15.3) and 0.1 years of projected life expectancy (18.3 vs. 18.2) favored neratinib after trastuzumab versus trastuzumab alone. The neratinib cost per QALY gained was $\$ 416,106$. At standard willingness-topay thresholds of $\$ 50,000, \$ 100,000$, and $\$ 200,000$ per QALY gained, neratinib has a probability of $2.8 \%, 16.7 \%$, and $33.9 \%$ of cost-effectiveness, respectively. The cost per QALY gained in a scenario analysis only including patients with hormone-receptor positive disease was $\$ 275,311$.
\end{abstract}

CONCLUSIONS: Based on 5-year data from ExteNET, neratinib following adjuvant trastuzumab is not projected to be cost-effective, even among those patients shown to derive the greatest clinical benefit. Future analyses should reassess the cost-effectiveness associated with neratinib treatment as trial data mature.

J Manag Care Spec Pharm. 2019;25(10):1133-39

Copyright @ 2019, Academy of Managed Care Pharmacy. All rights reserved.

\section{What is already known about this subject}

Neratinib after adjuvant trastuzumab significantly improves disease-free survival (DFS) in women with human epidermal growth factor receptor 2 (HER2)-positive breast cancer, but the median absolute DFS gain is only 1.3 months.

There has been much controversy in the clinical and lay media as to whether minimal gains in DFS are outweighed by the substantial cost and significant side effect profile.

\section{What this study adds}

This is the first independent assessment of the value of neratinib after adjuvant trastuzumab.

This study found that neratinib is not projected to be cost-effective, even among patients who derived the most clinical benefit. Study findings are important because they can help guide policy decisions and clinical guidelines

1 pproximately $15 \%-25 \%$ of breast cancers in the United States overexpress or have amplification of human epidermal growth factor receptor 2 (HER2), ${ }^{1-3}$ an adverse prognostic factor associated with increased rate of recurrence and significantly worse 5-year breast cancer-specific survival compared with HER2-negative disease (68\% vs. 96\%). ${ }^{4}$ Targeted therapy, such as trastuzumab in combination with chemotherapy, has altered the natural history of the disease, decreasing the rate of disease progression by approximately 25\%-40\%.-7 However, despite targeted therapy, approximately 20\%-30\% of early-stage patients will experience a recurrence within 10 years after diagnosis, two thirds of which is distant disease. ${ }^{5,7}$ Ten-year disease-free survival (DFS) after trastuzumab, while significantly better than observation alone, remains at only $69 \%-74 \% .^{5,7}$ This finding illustrates the potential role for additional therapies following adjuvant trastuzumab.

Ongoing efforts to further improve DFS in early-stage HER2positive disease have led to several phase III clinical trials evaluating additional targeted therapies after or in conjunction with adjuvant trastuzumab. ${ }^{8-10}$ The Adjuvant Lapatinib and/or Trastuzumab Treatment Optimization trial did not find any additional benefit associated with adding lapatinib, ${ }^{8}$ whereas the APHINITY and ExteNET trials demonstrated modest benefits in DFS with the addition of pertuzumab or neratinib, respectively. ${ }^{9}$ Subsequently, pertuzumab and neratinib were approved by the U.S. Food and Drug Administration. ${ }^{11,12}$

The approval of neratinib was controversial due to concerns about efficacy and drug safety. Critics highlighted the relatively small absolute margin of improvement in DFS (5-year DFS $90 \%$ vs. 88\%) and the severity of the side effects, with $40 \%$ of women experiencing grade 3 diarrhea. ${ }^{10}$ Compounding these issues, neratinib was projected to cost $\$ 10,500$ a month (\$126,000 per year), ${ }^{13}$ far exceeding the mean treatment cost 


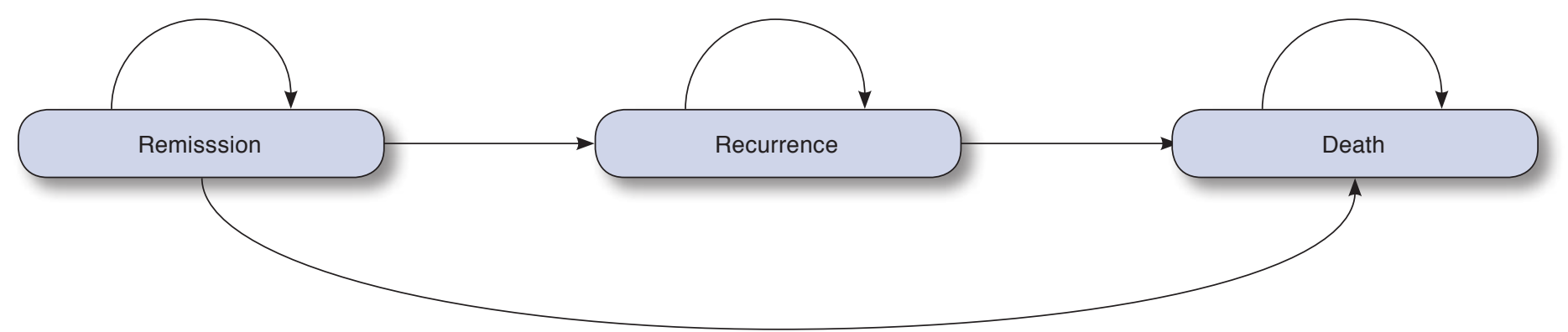

for stage I/II breast cancer in the first year after diagnosis $(\$ 82,121){ }^{14}$

As such, there is a need to understand whether therapies producing marginal gains in DFS, which have not been shown to be reliable predictors of overall survival (OS) ${ }^{15}$ can continue to be supported in an era of burgeoning health care expenditures. The objective of this analysis was to evaluate the cost per quality-adjusted life-year (QALY) gained of neratinib for early-stage HER2-positive breast cancer after adjuvant trastuzumab based on the ExteNET trial. Findings will aid in policy development and clinical decision making in the treatment of patients with HER2-positive breast cancer.

\section{Methods}

\section{Model Overview}

To conduct this cost-effectiveness analysis, we developed a Markov state-transition model composed of 3 health states: (1) remission/no evidence of disease, (2) recurrence, and (3) death (Figure 1). We estimated survival, QALYs, and direct medical expenditure in women with stage I-III HER2-positive breast cancer after 1-2 years of adjuvant trastuzumab followed by either observation or 1 year of neratinib. The model tracked cohorts in 1-month cycles over a lifetime horizon of up to 50 years of follow-up. Costs and outcomes were discounted at 3\%, following the recommendation of the Panel on CostEffectiveness in Health and Medicine. ${ }^{16}$ The analysis was performed from a health care payer perspective.

\section{Patient Population}

The base case analysis evaluated a cohort of women with a mean starting age of 50 years, reflecting the ExteNET patient population. ${ }^{10}$ Eligible women were aged $\geq 18$ years with no evidence of recurrent disease. Patients were required to have completed trastuzumab therapy less than 2 years before randomization and to have an Eastern Cooperative Oncology Group performance status of 0 or 1, normal organ function, and normal left ventricular ejection fraction. Patients with clinically significant cardiac, gastrointestinal, or psychiatric comorbidities were excluded, as were patients unable to swallow oral medications. Estimates of disease recurrence were based on 5-year follow-up data. ${ }^{17}$

\section{Direct Medical Expenditure}

Estimated costs of breast cancer recurrence and remission were based on peer-reviewed literature, ${ }^{18,19}$ as presented in a costeffectiveness analysis comparing adjuvant trastuzumab regimens (Table 1).$^{20}$ The cost of breast cancer recurrence included chemotherapy, supportive and end-of-life care, and up to 9 months of trastuzumab therapy. The monthly cost of neratinib was based on the wholesale acquisition cost. Costs of grade 3 and grade 4 adverse events (AEs) were derived from discharge data from the Nationwide Inpatient Sample and the Healthcare Cost and Utilization Project. ${ }^{21}$ All costs were inflation-adjusted to 2018 U.S. dollars using the medical care component of the Consumer Price Index. ${ }^{22}$

\section{Disease-Free and Overall Survival}

Survival outcomes for the 5 years following initiation of treatment with neratinib were based on follow-up data from ExteNET, the only phase III clinical trial studying outcomes associated with neratinib treatment (Table 1) ${ }^{17}$ To model a conservative effect of neratinib, it was assumed that the relative effect of neratinib (modeled as the DFS hazard ratio [HR]) was limited to the 5 years of follow-up. DFS after 60 months was based on annualized HRs from long-term follow-up of the HERceptin Adjuvant (HERA) trial 5 — a study that included early stage HER2-positive patients randomized to receive observation or adjuvant trastuzumab for 1 year or 2 years following completion of locoregional therapy and neo- or adjuvant chemotherapy. Because long-term follow-up data suggest that the risk of relapse in treated HER2-positive breast cancer is minimal beyond 10 years, ${ }^{23}$ we assumed that no recurrences occurred among women who were disease-free after 120 months.

In women without recurrence, the probability of death was derived from the most recent (2014) age-specific mortality estimates from the Social Security Administration. ${ }^{24}$ After 


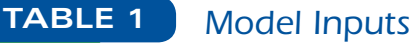

\begin{tabular}{|c|c|c|c|c|c|}
\hline Input Parameters & Point Estimate & Low Value & High Value & Distribution & Reference \\
\hline \multicolumn{6}{|l|}{ Costs (undiscounted), \$ } \\
\hline \multicolumn{6}{|l|}{ Well state, monthly } \\
\hline $50-54$ years & 258.69 & 238.28 & 291.23 & Normal & 20 \\
\hline $55-64$ years & 303.96 & 314.50 & 342.19 & Normal & 20 \\
\hline $65-74$ years & 386.74 & 357.26 & 435.38 & Normal & 20 \\
\hline$\geq 75$ years & 381.57 & 344.90 & 429.56 & Normal & 20 \\
\hline Breast cancer recurrence, monthly & $4,835.85$ & $4,546.29$ & $5,556.57$ & Normal & 20 \\
\hline \multicolumn{6}{|l|}{ Breast cancer end of life care, monthly } \\
\hline$<65$ years & $109,840.27$ & $108,392.13$ & $120,824.30$ & Normal & 46 \\
\hline$\geq 65$ years & $73,226.85$ & $75,000.96$ & $80,549.54$ & Normal & 46 \\
\hline Neratinib, monthly & $11,445.00$ & $10,300.50$ & $12,589.50$ & Normal & 47 \\
\hline Diarrhea, one-time cost & $7,927.53$ & $7,134.78$ & $8,720.28$ & Normal & 21 \\
\hline Abdominal pain, one-time cost & $6,471.30$ & $5,824.17$ & $7,118.43$ & Normal & 21 \\
\hline Nausea, one-time cost & $5,185.89$ & $4,666.40$ & $5,703.38$ & Normal & 21 \\
\hline Vomiting, one-time cost & $6,471.30$ & $5,824.17$ & $7,118.43$ & Normal & 21 \\
\hline Fatigue, one-time cost & $7,782.12$ & $7,003.91$ & $8,560.33$ & Normal & 21 \\
\hline Two outpatient clinic visits, one-time cost & 203.91 & 183.52 & 224.30 & Normal & 48 \\
\hline Recurrence, first 5 years (hazard ratio) & 0.73 & 0.46 & 0.84 & Beta & 17 \\
\hline \multicolumn{6}{|l|}{ Recurrence after 5 years (yearly hazard rates) } \\
\hline Years 5-6 & 0.019 & 0.013 & 0.029 & Beta & 5 \\
\hline Years 6-7 & 0.027 & 0.019 & 0.038 & Beta & 5 \\
\hline Years 7-8 & 0.015 & 0.010 & 0.025 & Beta & 5 \\
\hline Years 8-9 & 0.011 & 0.006 & 0.020 & Beta & 5 \\
\hline Years $9-10$ & 0.014 & 0.010 & 0.025 & Beta & 5 \\
\hline Years 10+ & 0.000 & 0.000 & 0.000 & Beta & Assumed \\
\hline Death due to breast cancer recurrence & 0.0272 & 0.022 & 0.033 & Beta & 49 \\
\hline Noncancer-related death & Age dependent & $+20 \%$ & $-20 \%$ & & 24 \\
\hline \multicolumn{6}{|c|}{ Grade 3/4 adverse events, neratinib (one-time probability) } \\
\hline Diarrhea & 0.40 & 0.320 & 0.480 & Beta & 10 \\
\hline Abdominal pain & 0.02 & 0.016 & 0.024 & Beta & 10 \\
\hline Nausea & 0.02 & 0.016 & 0.025 & Beta & 10 \\
\hline Vomiting & 0.03 & 0.024 & 0.036 & Beta & 10 \\
\hline Fatigue & 0.02 & 0.016 & 0.024 & Beta & 10 \\
\hline \multicolumn{6}{|c|}{ Grade 3/4 adverse events, standard care (one-time probability) } \\
\hline Diarrhea & 0.02 & 0.016 & 0.024 & Beta & 10 \\
\hline Abdominal pain & 0.00 & 0.000 & 0.000 & Beta & 10 \\
\hline Nausea & 0.01 & 0.008 & 0.012 & Beta & 10 \\
\hline Vomiting & 0.00 & 0.000 & 0.000 & Beta & 10 \\
\hline Fatigue & 0.00 & 0.000 & 0.000 & Beta & 10 \\
\hline \multicolumn{6}{|l|}{ Health state utilities } \\
\hline \multicolumn{6}{|l|}{ Remission, neratinib } \\
\hline Baseline & 0.863 & 0.858 & 0.868 & Beta & 25 \\
\hline Month 1-2 & 0.841 & 0.836 & 0.846 & Beta & 25 \\
\hline Month 3-5 & 0.841 & 0.836 & 0.847 & Beta & 25 \\
\hline Month 6-8 & 0.841 & 0.835 & 0.848 & Beta & 25 \\
\hline Month 9-11 & 0.849 & 0.843 & 0.856 & Beta & 25 \\
\hline Month 12 & 0.857 & 0.851 & 0.865 & Beta & 25 \\
\hline \multicolumn{6}{|l|}{ Remission, placebo } \\
\hline Baseline & 0.861 & 0.856 & 0.866 & Beta & 25 \\
\hline Month 1-2 & 0.863 & 0.858 & 0.869 & Beta & 25 \\
\hline Month 3-5 & 0.841 & 0.841 & 0.851 & Beta & 25 \\
\hline Month 6-8 & 0.846 & 0.835 & 0.847 & Beta & 25 \\
\hline Month 9-11 & 0.841 & 0.840 & 0.851 & Beta & 25 \\
\hline Month 12 & 0.849 & 0.853 & 0.857 & Beta & 25 \\
\hline Remission, no drug (after 12 months) & 0.850 & 0.680 & 1.000 & Beta & 25 \\
\hline Disease progression & 0.600 & 0.400 & 0.700 & Beta & 28 \\
\hline
\end{tabular}


Disease-free survival hazard ratio (range: 0.46-0.84)

Annual discount rate (range: 0.0-0.5)

Probability of recurrence, 9-10 years (range: 0.051\%-0.17\%)

Health state utility for disease progression (range: 0.4-0.7)

Cost of neratinib (range: $\$ 10,300.50-\$ 12,589.50)$

Probability of breast cancer death (range: $2.2 \%-3.3 \%$ )

Cost of recurrence (range: $\$ 4,546.29-\$ 5,556.57$ )

Probability of diarrhea with neratinib (range: 0.32-0.48)

Cost of diarrhea (range: $\$ 7,134.78-\$ 8,720.28$ )

Cost of breast cancer death, $\geq 65$ (range: $\$ 65,904.17-\$ 80,549.54$ )

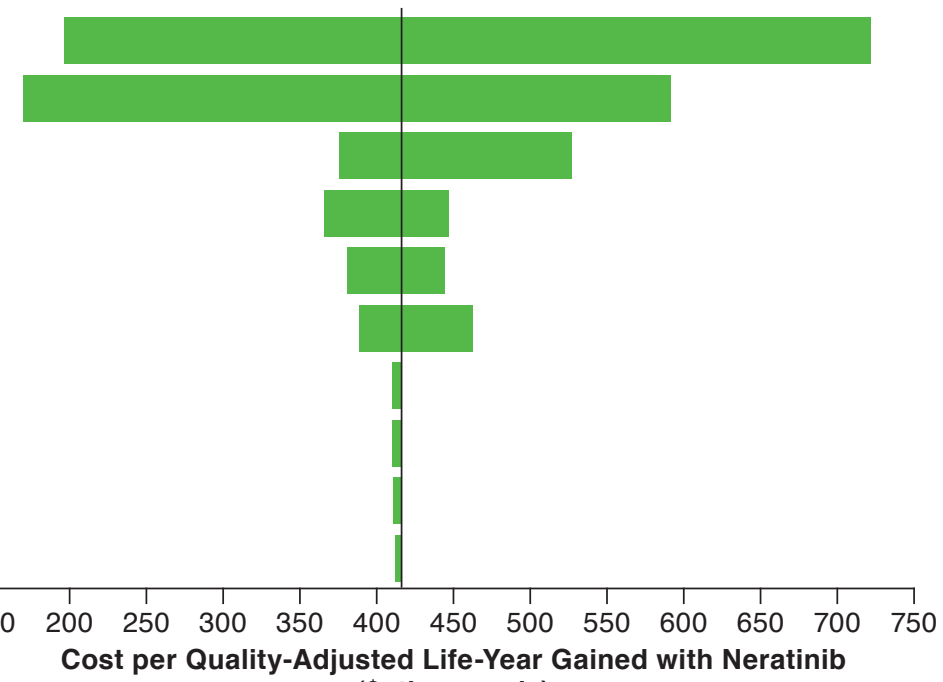

(\$, thousands)

recurrence, the mortality rate was derived from follow-up observations in the HERA trial. ${ }^{5}$

\section{Health-Related Quality of Life}

We incorporated estimates of health state utility for neratinib treatment, remission, and disease progression. Utilities were derived from health-related quality of life questionnaires from the ExteNET trial. ${ }^{25}$ Patients completed EuroQuol-5D questionnaires at baseline and months $1,3,6,9$, and 12 . Of the 2,840 patients randomized to study treatment, 2,427 (85.5\%) completed questionnaires at baseline and at least 1 post-baseline time point $(1,186$ in the neratinib arm, 1,241 in the placebo arm) ${ }^{25}$ Remission after 12 months was assigned a utility of $0.85,{ }^{26}$ and disease recurrence was assigned a utility of 0.6 based on previous publications. ${ }^{27,28}$

\section{Adverse Events}

We modeled the probability of grade 3 and grade 4 AEs that occurred in at least 2\% of the ExteNET trial population, including diarrhea, abdominal pain, nausea, vomiting, and fatigue. ${ }^{10}$ The model included one-time probabilities of AEs for each treatment arm, based on reported occurrences in ExteNET (Table 1). Any effect of AEs on quality of life was assumed to be captured by the health state utilities derived from the ExteNET trial. Thus, no utility decrements were assigned to individual AEs.

\section{Analyses}

We conducted a probabilistic analysis with 5,000 simulations to evaluate the cost per QALY gained and the range of incremental outcomes given uncertainty in all parameters. The base case HR for recurrence was varied within the 95\% confidence intervals [CIs] reported in the ExteNET trial, ${ }^{17}$ using a lognormal distribution. Costs were varied $\pm 10 \%$ using normal distributions. Ranges for health state utilities and transition probabilities were varied within $20 \%$ using a beta distribution or based on published values when available.

We also conducted a one-way sensitivity analysis to evaluate the most influential model inputs. The ranges of costs per QALY gained associated with uncertainty in each input are presented in the form of a tornado diagram (Figure 2).

\section{Scenario and Threshold Analyses}

Because prophylactic treatment with loperamide has been shown to diminish the duration and severity of neratinibassociated diarrhea, ${ }^{29}$ we considered an alternative scenario in which diarrhea was treated during 2 outpatient clinic visits rather than during inpatient visits. We also did this for nausea, vomiting, fatigue, and abdominal pain. Additionally, neratinib conferred more clinical benefit to individuals with hormone receptor-positive disease (hormone receptor-positive invasive DFS HR $=0.60,95 \% \mathrm{CI}=0.43-0.83$ vs. hormone receptor-negative $\mathrm{HR}=0.95,95 \% \mathrm{CI}=0.66-1.35$ ), so we constructed an alternative scenario in which the treatment population consisted only of hormone receptor positive patients.

To account for limited follow-up from the ExteNET trial, we explored a scenario in which the effect of neratinib persisted through 120 months, after which no recurrences were modeled. We also performed a threshold analysis to assess the price at which neratinib would be cost-effective. 


\begin{tabular}{l|c|c|c}
\hline \multicolumn{1}{c|}{ TABLE 2 } & Base Case Cost-Effectiveness Results \\
\hline & Neratinib & $\begin{array}{c}\text { Observation } \\
\text { After } \\
\text { Trastuzumab }\end{array}$ & Difference \\
\hline Results & 317,619 & 152,812 & 164,806 \\
\hline Cost, \$ & 18.31 & 18.17 & 0.14 \\
\hline Life-years & 15.67 & 15.28 & 0.40 \\
\hline Quality-adjusted life-years & - & - & $1,183,223$ \\
\hline Cost per life-year, \$ & - & - & 416,106 \\
\hline $\begin{array}{l}\text { Cost per quality-adjusted } \\
\text { life-year, \$ }\end{array}$ & & & \\
\hline
\end{tabular}

\section{Results}

\section{Base Case}

In the base case, neratinib treatment after trastuzumab yielded 15.67 QALYs at a cost of $\$ 317,619$ compared with 15.28 QALYs at a cost of $\$ 152,812$ with trastuzumab alone (Table 2). The addition of neratinib after trastuzumab produced an estimated life expectancy of 18.31 years compared with 18.17 years for trastuzumab alone, resulting in a difference of 0.14 life-years (LY). The difference in cost was $\$ 164,806$. The cost per QALY gained was $\$ 416,106$, and the cost per LY gained was $\$ 1,183,223$. At typical willingness-to-pay thresholds of $\$ 50,000$ per QALY, $\$ 100,000$ per QALY, and $\$ 200,000$ per QALY, ${ }^{30}$ the probability for neratinib to be considered costeffective is limited to $2.8 \%, 16.7 \%$, and $33.9 \%$, respectively (Figure 3).

\section{Sensitivity Analyses}

A one-way sensitivity analysis demonstrated that the results were most sensitive to variations in the DFS HR, the annual discount rate, and the probability of recurrence between 9 and 10 years (Figure 2 ).

Substituting the cost of 2 outpatient visits with inpatient care for treatment of AEs decreased costs to $\$ 313,965$ for neratinib therapy and $\$ 152,608$ for observation after trastuzumab. The resulting cost per QALY gained was $\$ 407,396$.

When the patient population was limited to only include patients with hormone-receptor positive disease, treatment with neratinib cost $\$ 317,369$ and produced 15.77 QALYs and 18.42 LYs. Observation after trastuzumab cost $\$ 154,530$ and produced 15.18 QALYs and 18.07 LYs. The resulting cost per QALY gained was $\$ 275,311$.

Extending the effect of neratinib to 120 months produced 15.91 QALYs and 18.57 LYs in the neratinib arm. The total cost of neratinib therapy decreased to $\$ 315,462$ with a cost per QALY gained of $\$ 255,438$.

To be cost-effective at a willingness-to-pay threshold of $\$ 150,000$ per QALY gained in the base case analysis, neratinib would need to cost $\$ 1,700$ per month. There was no price at which neratinib was cost-effective at a willingness-to-pay threshold of $\$ 100,000$ per QALY gained.

\section{Discussion}

Our analysis shows that adding neratinib after 1-2 years of adjuvant trastuzumab compared with trastuzumab alone in women with stage I-III HER2-positive breast cancer marginally improved life expectancy (18.31 years vs. 18.17 years) but did so at substantial additional cost ( $\$ 317,619$ vs. $\$ 152,812)$. At a base case cost of $\$ 416,106$ per QALY gained, neratinib is not cost-effective at implied willingness-to-pay thresholds for cancer treatment in the United States., ${ }^{31,32}$ To be considered a cost-effective use of scarce resources at a willingness-to-pay threshold of $\$ 150,000$ per QALY gained, the price of neratinib would need to be reduced by $85 \%(\$ 9,745)$. Patients with hormone-receptor positive breast cancer derived more clinical benefit from neratinib than those with hormone-receptor negative disease through 5 years of follow-up, ${ }^{17}$ but even among these patients, the clinical benefit achieved does not justify the high cost of neratinib, since the resulting cost per QALY gained of $\$ 275,311$ still exceeds willingness-to-pay thresholds. Even with additional sensitivity analyses that accounted for improved management of AEs (e.g., loperamide to decrease diarrhea) and limited the population to patients who received the greatest clinical benefit, we were unable to identify a scenario in which neratinib would be cost-effective.

Since the introduction of trastuzumab for HER-2 positive breast cancer, survival has improved significantly., ${ }^{5,73,34}$ Results from several cost-effectiveness analyses indicate that trastuzumab is cost-effective, ${ }^{20,27,35,36}$ with costs per QALY gained ranging from $\$ 18,970$ per QALY $^{35}$ to $\$ 39,982$ per QALY. $^{20}$ Trastuzumab is a high-value cancer treatment that substantially improves clinical outcomes at an acceptable price. New additive adjuvant therapies must demonstrate similar cost-effectiveness to add value to current HER2-targeted breast cancer treatment. In this context, neratinib appears to be low value.

Interestingly, the results of our study are comparable to findings from cost-effectiveness analyses of pertuzumab, a frequently used HER2-targeted adjuvant therapy. Although there are no U.S.-based cost-effectiveness studies for the addition of pertuzumab to trastuzumab in early-stage breast cancer, in a cost-effectiveness analysis of dual-blockade pertuzumab and trastuzumab versus trastuzumab alone among metastatic breast cancer patients enrolled in CLEOPATRA, pertuzumab had a cost per QALY gained of $\$ 472,668 .{ }^{37}$ This highlights the need for higher value drugs to be developed in this disease area.

\section{Limitations}

There are several limitations to this study. For the base case analysis, we assumed the disease control effect of neratinib was limited to the 5 years of follow-up data available from the ExteNET trial. To address this limitation, we performed an analysis extending the effect of neratinib to 120 months and found that even with extended therapeutic benefit, the cost per QALY gained was higher than standard willingness-to-pay 


\section{FIGURE 3 Cost-Effectiveness Acceptability Curve for Treatment with Neratinib in the Base Case}

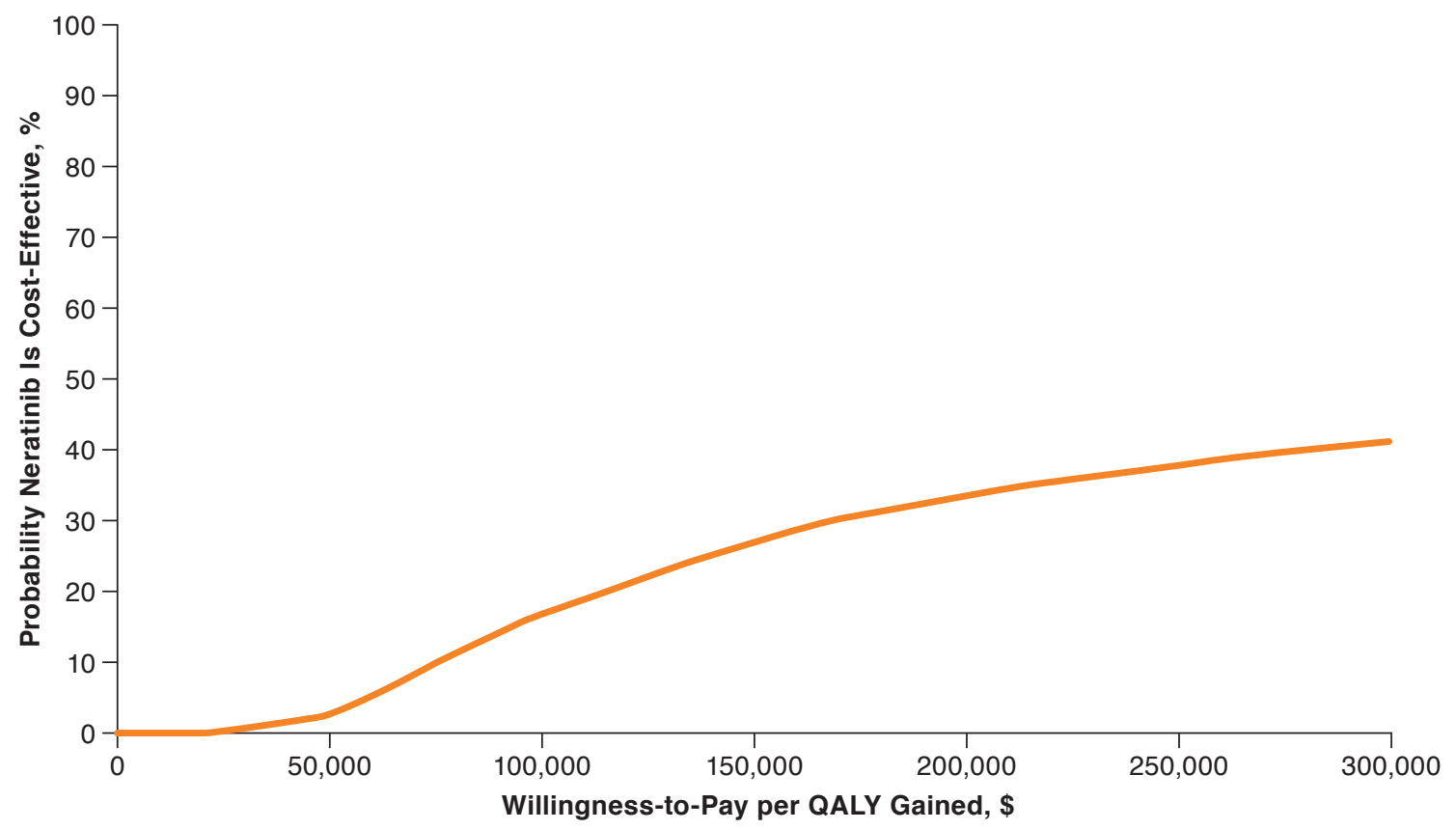

QALY= quality-adjusted life-year.

thresholds. Additionally, because OS data were not available from the ExteNET trial, we used OS data from the HERA trial. This was based on the assumption that, while neratinib reduces the risk of recurrence, it is unlikely to reduce the risk of death once recurrence occurs. Finally, because there is no long-term clinical data for neratinib use, our model only includes AEs that occurred during treatment. If neratinib increases the risk of future negative outcomes such as cardiac events, then that would result in an increased cost per QALY gained.

\section{Conclusions}

Over the past 2 decades, the price of cancer drugs has spiraled upward. ${ }^{38,39}$ This increase in treatment cost presents a considerable financial burden to patients, creating barriers to access and negatively affecting patient outcomes. ${ }^{40}$ This disproportionately affects patients of lower socioeconomic status, furthering health disparities. ${ }^{40}$ To combat skyrocketing prices, emphasis has been placed on "value-based" pricing, ${ }^{41}$ a method for pricing drugs based on how much the drug improves outcomes. However, defining the "value" of a drug is a complex task, since it requires assigning limits to the price of life. To assist with this, the American Society of Clinical Oncology and the European Society for Medical Oncology have developed frameworks to evaluate the clinical benefit of oncology drugs. ${ }^{42,43}$ Studies using these tools have revealed that a substantial proportion of recent therapies fail to meet thresholds for meaningful clinical benefit, 44,45 underscoring the need to develop drugs that provide substantial clinical benefit over and above the standard of care.

Although treatment with neratinib significantly, though marginally, improves DFS, there is still no data available on the long-term clinical benefit or OS. Until further evidence is available, neratinib should be reserved for high-risk patients with hormone-receptor positive disease. The high cost of neratinib coupled with the clinical benefit it currently confers prevents it from being recommended for use in all HER2-positive patients.

\section{Authors}

NAOMI R.M. SCHWARTZ, MPH, The Comparative Health Outcomes, Policy, and Economics (CHOICE) Institute, University of Washington, Seattle; MEGHAN R. FLANAGAN, MD, MPH, Department of Surgery, University of Washington School of Medicine, and Fred Hutchinson Cancer Research Center, Seattle, Washington; JOSEPH B. BABIGUMIRA, PhD, MS, Global Health, University of Washington, Seattle; LOTTE M. STEUTEN, PhD, MSc, Office of Health Economics, London, United Kingdom, and Fred Hutchinson Cancer Research Center, Seattle, Washington; and JOSHUA A. ROTH, PhD, MHA, Fred Hutchinson Cancer Research Center, Seattle, Washington.

AUTHOR CORRESPONDENCE: Naomi R.M. Schwartz, MPH, CHOICE Institute, University of Washington, 1959 N.E. Pacific St., H362 Health Sciences Bldg., Seattle, WA 98195. Tel. : 206.295.0633; E-mail:nschwa@uw.edu. 


\section{DISCLOSURES}

No outside funding supported this study. Roth reports consulting fees from Genentech. Steuten reports grants from AstraZeneca, EMD Serono, and Genomic Health, along with personal fees from Agendia, unrelated to this study. The other authors have no conflicts of interest in connection with this study.

\section{REFERENCES}

1. Cronin KA, Harlan LC, Dodd KW, Abrams JS, Ballard-Barbash R. Population-based estimate of the prevalence of HER-2 positive breast cancer tumors for early stage patients in the U.S. Cancer Invest. 2010;28(9):963-68

2. Slamon DJ, Clark GM, Wong SG, et al. Human breast cancer: correlation of relapse and survival with amplification of the HER-2/neu oncogene. Science, 1987:235 (4785):177-82.

3. Slamon DJ, Godolphin W, Jones LA, et al. Studies of the HER-2/neu proto-oncogene in human breast and ovarian cancer. Science. 1989;244(4905):707-12

4. Tovey SM, Brown S, Doughty JC, et al. Poor survival outcomes in HER2-positive breast cancer patients with low-grade, node-negative tumours. $\mathrm{Br} J$ Cancer. $2009 \cdot 100(5) \cdot 680-83$

5. Cameron D, Piccart-Gebhart MJ, Gelber RD, et al. 11 years' follow-up of trastuzumab after adjuvant chemotherapy in HER2-positive early breast cancer: final analysis of the HERceptin Adjuvant (HERA) trial. Lancet. 2017;389(10075):1195-205.

6. Slamon D, Eiermann W, Robert N, et al. Abstract S5-04: Ten year follow-up of BCIRG-006 comparing doxorubicin plus cyclophosphamide followed by docetaxel $(\mathrm{AC} \rightarrow \mathrm{T})$ with doxorubicin plus cyclophosphamide followed by docetaxel and trastuzumab $(\mathrm{AC} \rightarrow \mathrm{TH})$ with docetaxel, carboplatin and trastuzumab (TCH) in HER2+ early breast cancer. Cancer Res. 2016;76 (4 Suppl):S5-04. Available at: http://cancerres. aacrjournals.org/content/76/4_Supplement/S5-04. Accessed July 15, 2019.

7. Perez EA, Romond EH, Suman VJ, et al. Trastuzumab plus adjuvant chemotherapy for human epidermal growth factor receptor 2-positive breast cancer: planned joint analysis of overall survival from NSABP B-31 and NCCTG N9831. J Clin Oncol 2014;32(33):3744-52

8. Moreno-Aspitia A, Holmes EM, Jackisch C, et al. Updated results from the phase III ALTTO trial (BIG 2-06; NCCTG (alliance) N063D) comparing one year of anti-HER2 therapy with lapatinib alone $(\mathrm{L})$, trastuzumab alone $(\mathrm{T})$, their sequence $(\mathrm{T} \rightarrow \mathrm{L})$ or their combination ( $\mathrm{L}+\mathrm{T}$ ) in the adjuvant treatment of HER2-positive early breast cancer [abstract]. J Clin Oncol. 2017;35(15 Suppl):502. Available at: https://ascopubs.org/doi/ abs/10.1200/JCO.2017.35.15 suppl.502. Accessed July 15, 2019.

9. Von Minckwitz G, Procter M, De Azambuja E, et al. Adjuvant pertuzumab and trastuzumab in early HER2-positive breast cancer. N Engl J Med. 2017;377(2):122-31.

10. Chan A, Delaloge S, Holmes FA, et al. Neratinib after trastuzumab-based adjuvant therapy in patients with HER2-positive breast cancer (ExteNET): a multicentre, randomised, double-blind, placebo-controlled, phase 3 trial. Lancet Oncol. 2016;17(3):367-77.

11. Blumenthal GM, Scher NS, Cortazar P, et al. First FDA approval of dual anti-HER2 regimen: pertuzumab in combination with trastuzumab and docetaxel for HER2positive metastatic breast cancer. Clin Cancer Res. 2013;19(18):4911-16.

12. Singh H, Walker AJ, Amiri-Kordestani L, et al. U.S. Food and Drug Administration approval: neratinib for the extended adjuvant treatment of early stage HER2-positive breast cancer. Clin Cancer Res. 2018;24(15):3486-91.

13. Weintraub A. Puma to price new cancer med Nerlynx at a higher-than-expected \$10k per month: report. FiercePharma. July 20, 2017. Available at: https://www. fiercepharma.com/financials/puma-s-breast-cancer-drug-nerlynx-to-command-10k-amonth-despite-concerns-report. Accessed July 16, 2019.

14. Blumen H, Fitch K, Polkus V. Comparison of treatment costs for breast cancer, by tumor stage and type of service. Am Health Drug Ben. 2016;9(1):23-32.

15. Rupp T, Zuckerman D. Quality of life, overall survival, and costs of cancer drugs approved based on surrogate endpoints. JAMA Intern Med. 2017;177(2):276-77.

16. Gold M. Panel on cost-effectiveness in health and medicine. Med Care. 1996;34(12): DS197-DS199. Available at: https://www.jstor.org/stable/3766373?seq=1\#page_scan_ tab_contents. Accessed July 16, 2019.

17. Martin M, Holmes FA, Ejlertsen B, et al. Neratinib after trastuzumab-based adjuvant therapy in HER2-positive breast cancer (ExteNET): 5-year analysis of a randomised, double-blind, placebo-controlled, phase 3 trial. Lancet Oncol. 2017;18(12):1688-700.

18. Berkowitz N, Gupta S, Silberman G. Estimates of the lifetime direct costs of treatment for metastatic breast cancer. Value Health. 2000;3(1):23-30.

19. Rao S, Kubisiak J, Gilden D. Cost of illness associated with metastatic breast cancer. Breast Cancer Res Treat. 2004;83(1):25-32.

20. Kurian AW, Thompson RN, Gaw AF, et al. A cost-effectiveness analysis of adjuvant trastuzumab regimens in early HER2/neu-positive breast cancer. J Clin Oncol. 2007;25(6):634-41

21. Agency for Healthcare Research and Quality. Healthcare Cost and Utilization Project (HCUP). National Inpatient Sample. Available at: https://hcupnet.ahrq. gov/\#setup. Accessed July 31, 2019

22. U.S. Bureau of Labor Statistics. Consumer Price Index databases. Available at: https://www.bls.gov/cpi/data.htm. Accessed July 31, 2019.
23. Colleoni M, Sun Z, Price KN, et al. Annual hazard rates of recurrence for breast cancer during 24 years of follow-up: results from the International Breast Cancer Study Group Trials I to V. J Clin Oncol. 2016;34(9):927-35

24. Social Security Administration. Actuarial life tables. Period life table, 2014. Available at: https://www.ssa.gov/oact/STATS/table4c6_2014.html. Accessed July 31, 2019 25. Delaloge S, Ye Y, Cella D, et al. 177P Effects of neratinib (N) on health-related quality of life (HRQOL) in early-stage HER2+ breast cancer (BC): longitudinal analyses from the phase III ExteNET trial [abstract]. Ann Oncol. 2017;28(Suppl 5):mdx362.027. Available at: https://academic.oup.com/annonc/article/28/suppl_5/mdx362.027/ 4108344. Accessed July 16, 2019.

26. Fryback DG, Dasbach EJ, Klein R, et al. The Beaver Dam Health Outcomes Study: initial catalog of health-state quality factors. Med Decis Making. 1993;13(2):89-102. 27. Garrison LP, Lubeck D, Lalla D, et al. Cost-effectiveness analysis of trastuzumab in the adjuvant setting for treatment of HER2-positive breast cancer. Cancer 2007;110(3):489-98

28. Hornberger J, Kerrigan M, Foutel V. Cost-effectiveness of trastuzumab (Herceptin) for treatment of metastatic breast cancer. Ann Oncol. 2002;13(Suppl 5):Abstract 185P. 29. Hurvitz S, Chan A, Iannotti N, et al. Effects of adding budesonide or colestipol to loperamide prophylaxis on neratinib-associated diarrhea in patients with HER2+ early-stage breast cancer: the control trial. Cancer Res. 2018;78(4 Suppl):Abstract nr P3-14-01. Available at: http://cancerres.aacrjournals.org/content/78/4_Supplement/ P3-14-01. Accessed July 16, 2019.

30. Neumann PJ, Cohen JT, Weinstein MC. Updating cost-effectiveness-the curious resilience of the \$50,000-per-QALY threshold. N Engl J Med. 2014;371(9):796-97.

31. Neumann PJ, Palmer JA, Nadler E, Fang C, Ubel P. Cancer therapy costs influence treatment: a national survey of oncologists. Health Aff (Millwood). 2010;29(1):196-202

32. Bedikian AY, Millward M, Pehamberger $\mathrm{H}$, et al. Bcl-2 antisense (oblimersen sodium) plus dacarbazine in patients with advanced melanoma: the Oblimersen Melanoma Study Group. J Clin Oncol. 2006;24(29):4738-45.

33. Murthy RK, Varma A, Mishra P, et al. Effect of adjuvant/neoadjuvant trastuzumab on clinical outcomes in patients with HER2-positive metastatic breast cancer. Cancer. 2014;120(13):1932-38

34. Mendes D, Alves C, Afonso N, et al. The benefit of HER2-targeted therapies on overall survival of patients with metastatic HER2-positive breast cancer-a systematic review. Breast Cancer Res. 2015;17:140.

35. Liberato NL, Marchetti M, Barosi G. Cost effectiveness of adjuvant trastuzumab in human epidermal growth factor receptor 2-positive breast cancer. J Clin Oncol. 2007:25(6):625-33.

36. Millar JA, Millward MJ. Cost effectiveness of trastuzumab in the adjuvant treatment of early breast cancer: a lifetime model. PharmacoEconomics. 2007;25(5):429-42 37. Durkee BY, Qian Y, Pollom EL, et al. Cost-effectiveness of pertuzumab in human epidermal growth factor receptor 2-positive metastatic breast cancer. J Clin Oncol. 2016;34(9):902-09.

38. Gordon N, Stemmer SM, Greenberg D, Goldstein DA. Trajectories of injectable cancer drug costs after launch in the United States. J Clin Oncol. 2018;36(4):319-25. 39. Light DW, Kantarjian H. Market spiral pricing of cancer drugs. Cancer. 2013;119(22):3900-02

40. Carrera PM, Kantarjian HM, Blinder VS. The financial burden and distress of patients with cancer: understanding and stepping-up action on the financial toxicity of cancer treatment. CA Cancer J Clin. 2018;68(2):153-65.

41. Kantarjian HM, Fojo T, Mathisen M, Zwelling LA. Cancer drugs in the United States: justum pretium-the just price. J Clin Oncol. 2013;31(28):3600-04 42. Schnipper LE, Davidson NE, Wollins DS, et al. American Society of Clinical Oncology statement: a conceptual framework to assess the value of cancer treatment options. J Clin Oncol. 2015;33(23):2563-77.

43. Cherny NI, Sullivan R, Dafni U, et al. A standardised, generic, validated approach to stratify the magnitude of clinical benefit that can be anticipated from anti-cancer therapies: the European Society for Medical Oncology Magnitude of Clinical Benefit Scale (ESMO-MCBS). Ann Oncol. 2015;26(8):1547-73.

44. Del Paggio JC, Azariah B, Sullivan R, et al. Do contemporary randomized controlled trials meet ESMO thresholds for meaningful clinical benefit? Ann Oncol. 2017;28(1):157-62.

45. Dreicer JJ, Mailankody S, Fahkrejahani F, Prasad V. Clinically meaningful benefit: real world use compared against the American and European guidelines. Blood Cancer J. 2017;7(12):645.

46. Yabroff KR, Lund J, Kepka D, Mariotto A. Economic burden of cancer in the US: estimates, projections, and future research. Cancer Epidemiol Biomarkers Prev. 2011;20(10):2006-14.

47. First Databank. FDB MedKnowledge. Database. Retrieved November 20, 2017. Available at: https://www.fdbhealth.com/solutions/medknowledge. Accessed July 16, 2019. 48. Centers for Medicare \& Medicaid Services. Medicare provider utilization and payment data: outpatient. 2016. Available at: https://www.cms.gov/Research-StatisticsData-and-Systems/Statistics-Trends-and-Reports/Medicare-Provider-Charge-Data/ Outpatient.html. Accessed July 16, 2019.

49. Purmonen TT, Pankalainen E, Turunen JH, Asseburg C, Martikainen JA. Shortcourse adjuvant trastuzumab therapy in early stage breast cancer in Finland: costeffectiveness and value of information analysis based on the 5-year follow-up results of the FinHer Trial. Acta Oncol. 2011;50(3):344-52. 